\title{
High throughput screening identifies disulfide isomerase DsbC as a very efficient partner for recombinant expression of small disulfide-rich proteins in $E$. coli
}

Hervé Nozach ${ }^{1 *}$, Carole Fruchart-Gaillard ${ }^{1}$, François Fenaille ${ }^{3}$, Fabrice Beau' ${ }^{1}$ Oscar Henrique Pereira Ramos ${ }^{1}$, Badreddine Douzi ${ }^{2}$, Natalie J Saez ${ }^{2}$, Mireille Moutiez ${ }^{1}$, Denis Servent ${ }^{1}$, Muriel Gondry ${ }^{1}$, Robert Thaï ${ }^{1}$,

Philippe Cuniasse ${ }^{1}$, Renaud Vincentellii ${ }^{2 \dagger}$ and Vincent Dive ${ }^{1 \dagger}$

\begin{abstract}
Background: Disulfide-rich proteins or DRPs are versatile bioactive compounds that encompass a wide variety of pharmacological, therapeutic, and/or biotechnological applications. Still, the production of DRPs in sufficient quantities is a major bottleneck for their complete structural or functional characterization. Recombinant expression of such small proteins containing multiple disulfide bonds in the bacteria $E$. coli is considered difficult and general methods and protocols, particularly on a high throughput scale, are limited.

Results: Here we report a high throughput screening approach that allowed the systematic investigation of the solubilizing and folding influence of twelve cytoplasmic partners on 28 DRPs in the strains BL21 (DE3) pLysS, Origami B (DE3) pLysS and SHuffle ${ }^{\oplus}$ T7 Express lysY (1008 conditions). The screening identified the conditions leading to the successful soluble expression of the 28 DRPs selected for the study. Amongst 336 conditions tested per bacterial strain, soluble expression was detected in 196 conditions using the strain BL21 (DE3) pLysS, whereas only 44 and 50 conditions for soluble expression were identified for the strains Origami B (DE3) pLysS and SHuffle ${ }^{\oplus}$ T7 Express lysY respectively. To assess the redox states of the DRPs, the solubility screen was coupled with mass spectrometry (MS) to determine the exact masses of the produced DRPs or fusion proteins. To validate the results obtained at analytical scale, several examples of proteins expressed and purified to a larger scale are presented along with their MS and functional characterization.

Conclusions: Our results show that the production of soluble and functional DRPs with cytoplasmic partners is possible in E. coli. In spite of its reducing cytoplasm, BL21 (DE3) pLysS is more efficient than the Origami B (DE3) pLysS and SHuffle ${ }^{\oplus}$ T7 Express lysY trXB/gor strains for the production of DRPs in fusion with solubilizing partners. However, our data suggest that oxidation of the proteins occurs ex vivo. Our protocols allow the production of a large diversity of DRPs using DsbC as a fusion partner, leading to pure active DRPs at milligram scale in many cases. These results open up new possibilities for the study and development of DRPs with therapeutic or biotechnological interest whose production was previously a limitation.
\end{abstract}

Keywords: High throughput screening, Recombinant expression, Disulfide bonds, Cytoplasm, Disulfide-rich, Proteins, Fusion protein, Escherichia coli, Spectrometry, Mass, Matrix-assisted laser desorption-ionization

\footnotetext{
* Correspondence: herve.nozach@cea.fr

${ }^{\dagger}$ Equal contributors

'CEA, iBiTec-S, Service d'Ingénierie Moléculaire des Protéines (SIMOPRO), CEA

Saclay, Gif sur Yvette F-91191, France

Full list of author information is available at the end of the article
} 


\section{Introduction}

Small proteins containing disulfide bonds are versatile bioactive compounds that possess important pharmacological, therapeutic, and/or biotechnological values. There is a growing interest in the use of small disulfide-rich proteins (DRPs) as therapeutics, as they have several advantages over traditional small molecule drugs due to their high affinity and selectivity [1]. Small reticulated proteins have many applications, such as ion channel blockers for severe chronic pain treatment [2], as scaffolds for the transfer of hotspot active sites of bigger or more complex proteins $[3,4]$, as antimicrobial and host-defense peptides [5] or molecular imaging agents [6]. In most cases, DRPs are composed of 20 to 120 residues and include between one to five disulfide bonds that are often crucial for activity and stability of these proteins. Oxidation of cysteine residues and proper disulfide pairing are indeed necessary for the correct spatial distribution of the key functional residues. Unfortunately, study and development of DRPs of therapeutic or biotechnological interest are still hampered by the difficulty to produce native and active proteins in sufficient amounts.

E. coli has many well-known advantages as a host for heterologous protein expression [7]. Various and complementary approaches have been described to produce native and soluble proteins in this bacterial host. In the past decade, several high throughput platforms have been used to identify optimal conditions for the soluble expression of proteins, notably by varying parameters such as fusion partners, strains or temperature [8-12]. Surprisingly, whereas several examples of successful expression of reticulated proteins in E. coli have been described [13-16], there is, to our knowledge, no study reporting parallel expression screening of many proteins containing various numbers of disulfide bonds.

Even if the production of various disulfide-bonded proteins in $E$. coli has previously been reported, expression of proteins with multiple disulfide bonds is still considered difficult [17]. As shown in vitro for the wellstudied Bovine Pancreatic Trypsin Inhibitor (BPTI), the folding of disulfide-bonded proteins is often acquired through the accumulation of disulfide intermediates $[18,19]$. For some disulfide-rich proteins, oxidative folding generates heterogeneous populations of intermediates containing native but also non-native disulfide-bonded species, which require isomerization to reach the nativelyfolded oxidized state $[20,21]$. Thus, proteins with disulfide bonds are especially prone to aggregation due to possible mispairing of cysteine residues or undesirable intermolecular disulfide bonds. When overexpressed in bacteria with strong promoters, recombinant proteins often tend to misfold and accumulate as insoluble aggregates or inclusion bodies [22]. In many cases, the difficulty in reaching native conformation increases with the number of cysteine residues due to the number of possible isoforms, but also with the complexity of disulfide bond patterns. Failure to reach a native and stable conformation results, in most cases, in either protein aggregation or proteolytic degradation [23].

In past years, many approaches have been developed to promote the formation of disulfide bonds and the native folding of disulfide-rich proteins [17]. Exporting the proteins to the E. coli oxidizing periplasm is an intuitive strategy $[24,25]$, as folding of proteins can be assisted by the disulfide bond formation system [26-28]. However, secretion of proteins to the periplasm often leads to low protein levels [28], probably because of the limited periplasmic volume combined with an insufficient capacity of the translocation machinery [29]. Because of these limitations, many strategies consider expression in the E. coli cytoplasm, even for proteins containing disulfide bonds. Oxidation of cysteine thiols in the reducing cytoplasm of wild-type $E$. coli is described as unfavorable for both thermodynamic and kinetic reasons $[17,23]$. To overcome this issue, engineered strains like Origami (DE3) pLysS with an oxidative cytoplasm were developed $[30,31]$. These strains contain deletions of both glutathione and thioredoxin reductase genes $\left(\right.$ gor, $\left.\operatorname{tr} x B^{-}\right)$along with an additional mutation in the peroxiredoxin gene $a h p C$ necessary to restore growth. Some studies indicate that these strains enhance the accumulation of oxidized proteins in the cytoplasm [17,32,33]. Several other engineered strains with altered reducing pathways are described to improve production levels of disulfide-bonded proteins $[34,35]$. The amount of oxidized protein can be further enhanced by co-expression of redox-active enzymes like thioredoxin (Trx), Trx mutants or DsbC in the cytoplasm of $\operatorname{trx} B^{-} /$gor strains [30,36]. Thioredoxin mutants are of particular interest because the two residues included in the dicysteine active site $(\mathrm{CxxC})$ are effecting their oxidoreductase activities. Indeed, mutating these two amino acids shifts the activity from a reductase to an oxidase in oxidative environments. These characteristics are described as very useful for the production of proteins with disulfide bonds [30,37].

In addition to those approaches for promoting the formation of disulfide bonds, the question of the solubilization of the protein of interest and its folding intermediates has to be addressed. Many fusion tags are described to enhance protein solubility in vivo [38-40]. Several proteins have been extensively used as fusion partners like glutathione S-transferase (GST) [41], maltose binding protein (MBP) [42], double Z-domain from staphylococcal protein A (ZZ) [43] or Gb1-domain from protein G (Gb1) [44]. Some other fusion partners not only have an important solubilizing effect but also redox properties, which could be beneficial for disulfide bond formation. From this perspective, thioredoxin is not only an oxidoreductase but 
also one of the most potent solubilizing partners available [45]. For the above purpose, the proteins DsbA and DsbC, although not as yet explored, could also be of interest as solubilizing partners for cytoplasmic expression, if expressed without their signal peptides. Indeed, disulfide isomerase DsbC retains a foldase and chaperonin activity when used in co-expression in the cytoplasm $[36,46]$. Nevertheless, even if several publications report the positive effect of fusion partners or use of specific strains on the recombinant expression of specific DRPs, no general study has yet explored the efficacy of these systems on a large diversity of DRPs in order to document general rules for this protein family.

Here we report the results of a high throughput screen for the soluble expression of small proteins with disulfide bonds in the E. coli cytoplasm. Given a set of DRPs; 28 different proteins of variable size (from 25 to 122 aa) with two to five disulfide bridges, the objective of this study was to identify the best fusion partners and strains to provide access to milligram amounts of oxidized and functional recombinant DRPs.

\section{Results}

\section{Study set up}

We have selected 28 targets (Additional file 1: Table S1 Data), representing six different folds (ICK, 3FT, Kunitz, Kazal type, $\alpha / \beta, 3_{10}$ helix). This set includes proteins for which functional tests are available to allow the assessment of the correct folding of the targets based on the preservation of their native binding properties. All these proteins have an even number of cysteine residues that are all involved in a disulfide bond. Twelve different fusion partners targeting the protein into the cytoplasm were selected in our study (Additional file 2: Table S2 Data). In every case a hexa-histidine (6HIS) tag was introduced to enable the downstream purification of the fusion proteins using immobilized-nickel affinity chromatography. A TEV (Tobacco Etch Virus) protease cleavage site (ENLYFQ/G) was introduced to enable removal of the fusion partner. In addition to the $6 \mathrm{HIS}$ affinity tag alone which serves as a reference, the 11 other fusion partners selected in our study include glutathione S-transferase (GST) [41], maltose binding protein (MBP) [42], double Z-domain from staphylococcal protein A (ZZ) [43], Gb1-domain from protein G (Gb1) [44], thioredoxin ( $\operatorname{Trx})$ and four of its active site mutants [30], the disulfide oxidoreductase DsbA and the disulfide isomerase DsbC from E. coli [47] without their signal peptides to allow cytoplasmic expression. The four mutants of Thioredoxin are Trx-A with a DsbA-like active site (CPHC), Trx-C with a DsbC-like active site (CGYC), Trx-G with a glutaredoxin-like active site (CPYC) and Trx-P with a PDI-like active site (CGHC). All expression vectors were constructed using Gateway cloning technology (Invitrogen) [48]. A schematic representation of each vector can be found in Figure 1. To benchmark the effect of the strains on the yield of DRPs, three different $E$. coli expression strains were used in this study. BL21 (DE3) pLysS with a reducing cytoplasm, the Origami B (DE3) pLysS with a more oxidizing cytoplasm and finally the SHuffle ${ }^{\oplus}$ T7 Express lysY which are similar to the Origami B (DE3) pLysS (oxidizing cytoplasm) but was engineered to express also cytoplasmic DsbC [36]. The screening scheme (Figure 2) is a slightly modified version of the high throughput protocols described in detail elsewhere $([9,49])$. The culture and expression

\section{(A) : 6HIS (no solubilizing partner)

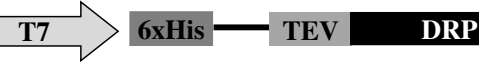

(B) : GST, MBP, ZZ, GB1 (fusions without redox properties)

T7 $>6 x H i s-2$ Fusion Partner

\section{(C) : Thioredoxins, DsbA, DsbC (fusions with redox properties)}

T7 $>$ Fusion Partner $-6 x H i s-$ TEV $\quad$ DRP

Figure 1 Design of the expression plasmids. All plasmids carry a T7 promoter/terminator, a 6HIS tag for nickel affinity purification, a Tobacco Etch Virus (TEV) cleavage site and a gene encoding a disulfide-rich protein (DRP). The 6 HIS tag is N-terminal for the 6 HIS plasmid (A) and for fusions without redox properties (B). The 6HIS tag is placed between the fusion partner and the TEV site for fusion with redox properties (C). Non-redox fusion partners are represented in white boxes while redox fusions are represented in blue. The same representation of the redox properties of the fusions appears throughout the figures. 


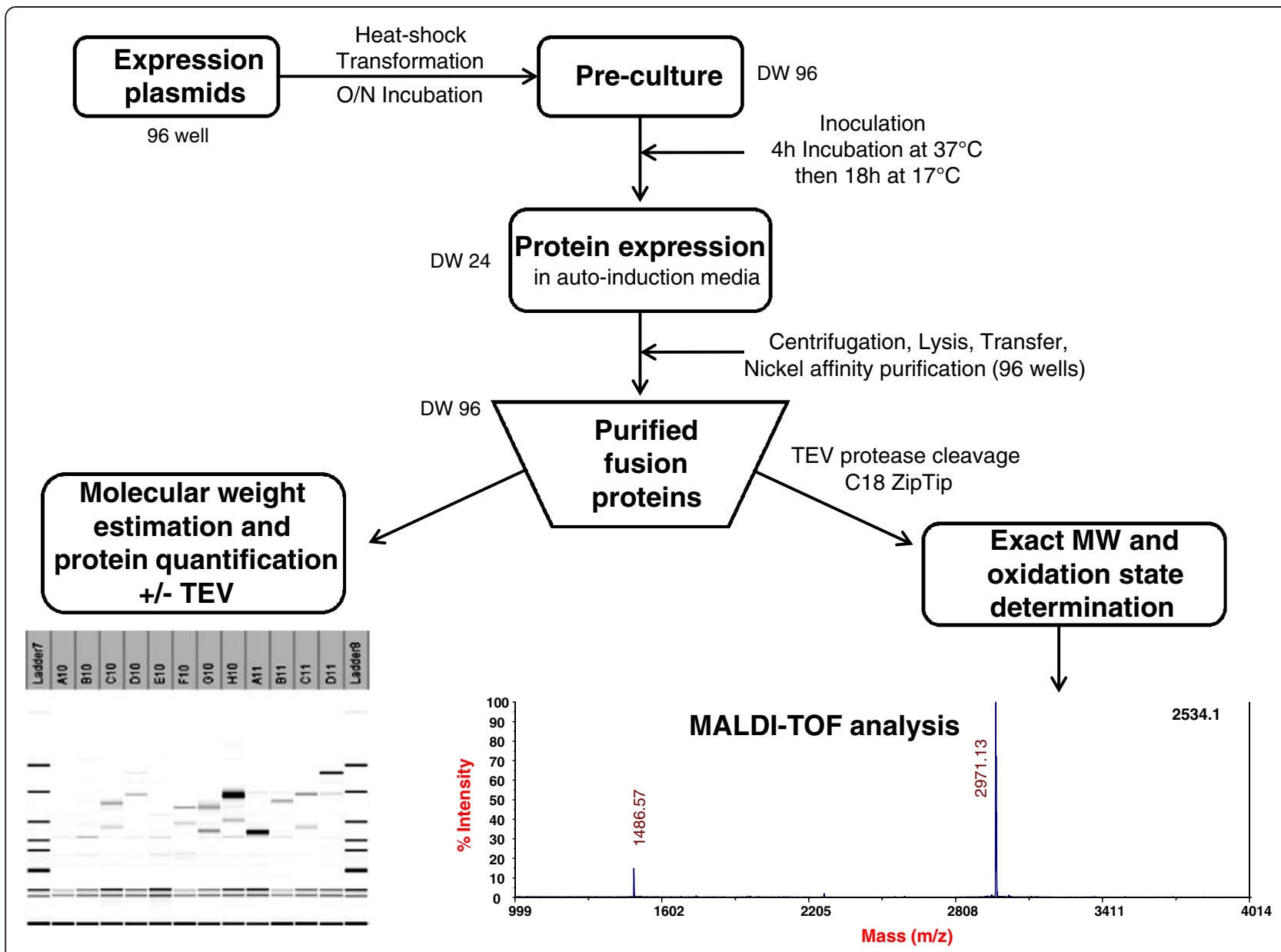

Figure 2 Schematic representation of the high throughput expression screening procedure. For details, see Materials and methods.

were performed in auto-induction media [50]. After nickel affinity purification, systematic analysis of the Labchip GXII electropherograms was performed to determine the concentration of the purified proteins and compare the apparent molecular weight of the purified fusion proteins with their expected theoretical molecular weight. After incubation with the protease TEV to remove the fusion partner, the cleavage was confirmed by analysis on the Labchip GXII and the accurate mass and oxidation state of each peptide were determined by mass spectrometry. Whenever possible, functional assays were performed to demonstrate the accuracy of their folding.

\section{Influence of the fusion partner and strains on soluble expression}

An exhaustive analysis of the 1008 conditions tested (28 proteins $\mathrm{x} 12$ fusion partners $\mathrm{x} 3$ strains) was performed in parallel (Figures 3, 4 and 5). The amounts of soluble fusion proteins were estimated after the nickel purification using the Caliper GX II semi quantitative method (See Materials and Methods). The experiments on the
1008 cultures were performed in duplicates. The soluble level was in good agreement between the two experiments and therefore the soluble yields were averaged between the two experiments and ranked according to four categories of soluble level: not detected, soluble from 0.1 to $10 \mathrm{mg} / \mathrm{L}, 10$ to $20 \mathrm{mg} / \mathrm{L}$ and greater than 20 $\mathrm{mg} / \mathrm{L}$. The limit of $20 \mathrm{mg} / \mathrm{L}$ was dictated by the volume of nickel beads used in the expression screening protocol $(50 \mu \mathrm{L})$; a volume suitable to detect low expressing proteins (>0.1 mg/L of culture with Caliper GX II detection) but saturated with soluble levels above $20 \mathrm{mg} / \mathrm{L}$ $[9,49]$. While the expression screening protocol used in this study gave a good indication of the soluble level and while it allowed the ranking of the impact of the fusion based on soluble yields, it was not designed to discriminate between several conditions that would all give soluble levels above $20 \mathrm{mg} / \mathrm{L}$. In this study, at preparative scale, the purified fusion proteins were sometimes above $200 \mathrm{mg} / \mathrm{L}$ (see "Functional characterization"). This prompted us to design a modified expression screening protocol (based on $200 \mu \mathrm{L}$ of beads [49]) that was validated on the high expressing fusions of this study and 
confirmed the ranking obtained with $50 \mu \mathrm{l}$ of beads (data not shown).

Out of 336 constructs in BL21 (DE3) pLysS, soluble expression of fusion proteins was observed in the milligram range of protein per liter of culture in 196 cases (58\%), with correct apparent molecular weights (Figure 3). Fusion with MBP, GST, DsbA and DsbC partners resulted in soluble expression of most of the DRPs (more than $85 \%$ for MBP, GST and DsbA and more than $95 \%$ for DsbC). Fusion with the different thioredoxins or $\mathrm{ZZ}$ was successful, but less effective, with soluble expression rates ranging from $40 \%$ to $70 \%$. Soluble expression was observed neither with the sole 6HIS tag nor with the fusion Gb1, where DRPs in fusion were often detected as inclusion bodies (data not shown). As shown in Figures 3 and 6, the four best solubilizing fusions (MBP, GST, DsbA and DsbC) not only gave access to a wider variety of soluble DRPs but also gave higher amounts of soluble protein for the 12 fusions tested. Many DRPs were expressed with significant expression levels when fused with those four partners. Specifically, 18 DRPs out of 28 were expressed with expression levels greater than $10 \mathrm{mg} / \mathrm{L}$ of fusion protein using DsbC as a fusion partner (Figure 6). This number is significantly higher with DsbC than for any of the other fusion partners tested (Figure 6).

In contrast, soluble expression was observed in only 44 conditions for the Origami B (DE3) pLysS strain (Figure 4) and in only 50 conditions in the strain SHuffle ${ }^{\circ} \mathrm{T} 7$ Express lysY (Figure 5) as compared to 196 with BL21 (DE3) pLysS strain (Figure 3). The estimated amounts of soluble fusion proteins obtained in both strains were almost always lower or in the best cases only similar to those produced in the same condition in BL21 (DE3) pLysS. For the Origami B (DE3) pLysS, the fusion with 


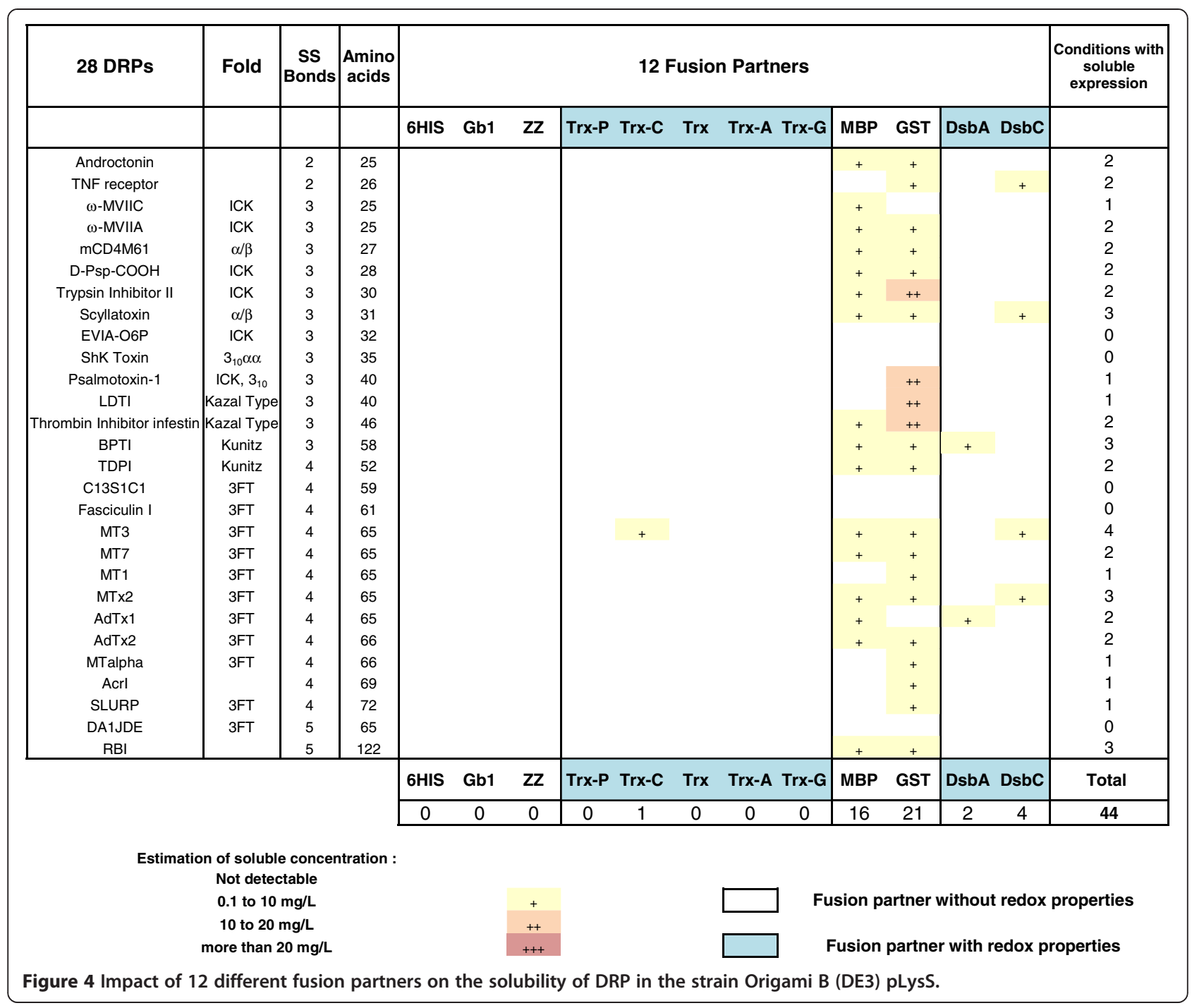

MBP or GST resulted in soluble expression for the majority of DRPs (57\% and $75 \%$ respectively) while soluble expression with thioredoxins, DsbA and DsbC were only observed in rare cases $(<1 \%,<10 \%$ and $<15 \%$ respectively). Out of the 44 soluble conditions, the soluble level was mainly achieved at very low concentrations (90\% of the cases are below $10 \mathrm{mg} / \mathrm{L}$ ) except in the cases of four GST fusions.

For the SHuffle ${ }^{\oplus}$ T7 Express lysY, the fusion with MBP was the only one allowing the soluble expression for a majority of DRPs (71\%). The successful soluble expression with $\mathrm{ZZ}$ and thioredoxins (below or around 5\%) was very rare while the fusion with DsbA and DsbC (40\%) was far from the same as cultures in BL21 (DE3) pLysS, but significantly better than in Origami B (DE3) pLysS. Out of the 50 soluble conditions, the soluble level was mainly achieved at very low concentrations $(78 \%$ of the cases are below $10 \mathrm{mg} / \mathrm{L})$. Most of the $22 \%$ that were expressed between 10 to $20 \mathrm{mg} / \mathrm{L}$ were expressed as MBP fusions.
In our set-up, the differences observed between BL21 (DE3) pLysS versus Origami B (DE3) pLysS and SHuffle T7 Express lysY were important, both in terms of number of soluble constructs and yields. In particular, of the 28 DRPs the soluble yield obtained with the Origami B (DE3) pLysS or the SHuffle ${ }^{\circledR}$ T7 Express lysY was never better than the yield of the equivalent condition in BL21 (DE3) pLysS. For these reasons, we have chosen to focus on the production of DRPs using only the strain BL21 (DE3) pLysS in the rest of the study.

\section{Influence of the fusion partner on oxidation}

Next, an extensive analysis of the oxidation state of the DRPs was performed by MALDI-TOF. As MALDI-TOF analyses with isotopic resolution are limited to very small proteins, only the 12 DRPs with a molecular mass lower than 6500 Da could be analyzed (Figure 7). All the constructs yielding soluble fusion proteins for those 12 DRPs in the strain BL21 (DE3) pLysS (96 out of 120) 
were submitted to TEV cleavage, desalting and MALDITOF analysis. Amongst these 96 constructs, the monoisotopic masses corresponding to the correctly oxidized species were detected in 81 cases (See Figure 7: conditions in green). In all analyses performed, the MALDI-TOF spectra matched the theoretical isotopic distribution for the DRP with fully oxidized cystines (see Figure 8A: experimental MALDI-TOF spectrum obtained for mCD4M61). The reduced forms of the proteins could not be detected (data not shown), probably due to aggregation or precipitation. Oxidized proteins were detected in a large majority of cases when fused to partners with redox activity (between 70 to $100 \%$ for thioredoxin and its mutants, $92 \%$ for DsbA and $100 \%$ for DsbC, see Figure 7). This percentage is lower when fusions have only a solubilizing role $(60 \%$ for $\mathrm{ZZ}$, $70 \%$ for MBP, $58 \%$ for GST). To access the oxidation state of DRPs larger than $6500 \mathrm{Da}$, we also analyzed selected DRPs in fusion with their solubilizing partner with an
LTQ-Orbitrap mass spectrometer. As DsbC was by far the best fusion partner among those investigated, the exact masses of 25 DsbC-DRP fusions were determined. These analyses demonstrated that out of these 25 samples, 17 fusion proteins had molecular masses in agreement with the expected masses considering fully oxidized cystine residues, with a mass difference between the observed and expected masses of less than $0.7 \mathrm{Da}$ on average (Additional file 3: Table S3).

As mass spectrometry analyses revealed that oxidized DRPs were detected in many cases, we tried to determine if the formation of disulfide bonds occurred in the cytoplasm of BL21 (DE3) pLysS or during the subsequent extraction and purification steps. To elucidate this question, we investigated the particular case of mCD4M61 produced in fusion with DsbC. A thiol-blocking agent, $\mathrm{N}$-ethyl maleimide (NEM) was introduced in the lysis buffer to prevent ex vivo oxidation. NEM readily reacts with free 


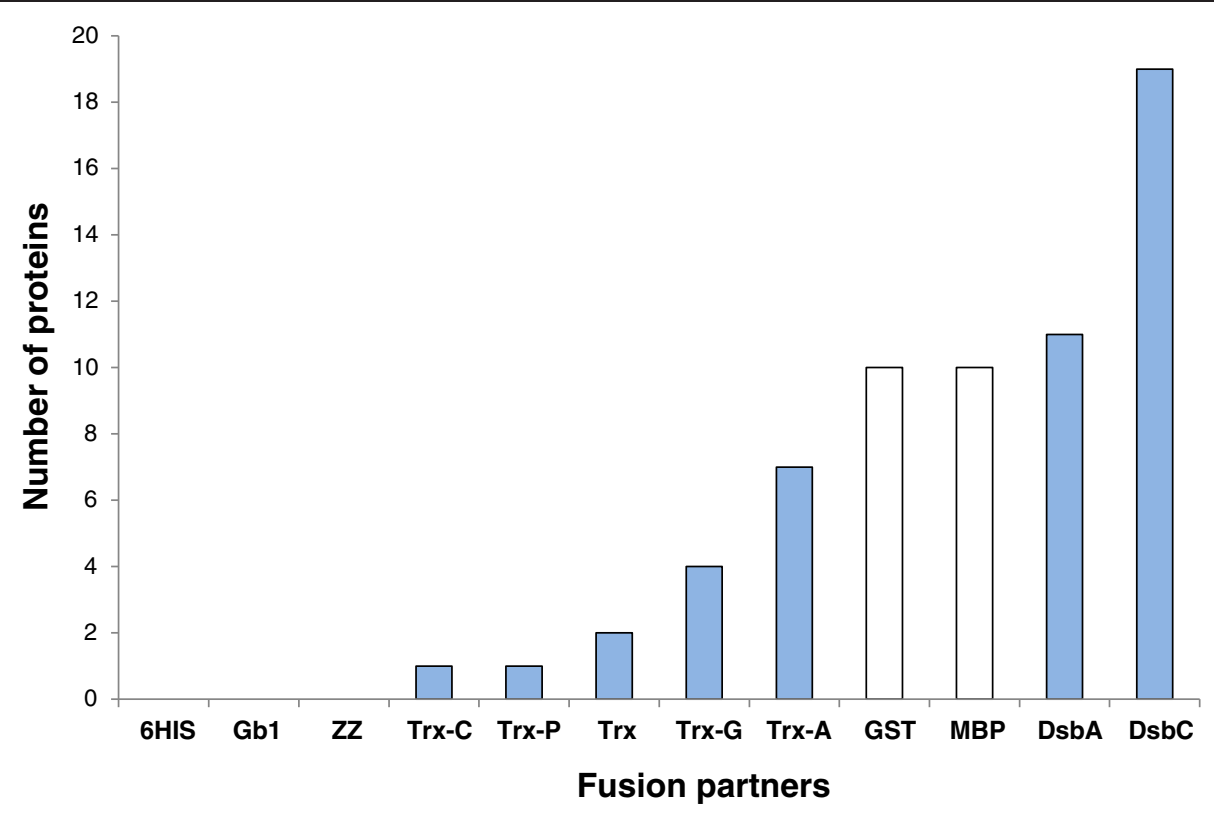

Figure 6 Impact of the fusion partner on the number of soluble proteins. White: Non-redox fusions. Blue: redox fusions. Bars represent the number of soluble DRPs produced above $10 \mathrm{mg} / \mathrm{L}$ of culture in fusion with the specified partner. The strain used is BL21 (DE3) pLysS.

cysteine residues, thus yielding a 125.13 Da mass increment per alkylated cysteine. However, as NEM does not react with cystines involved in disulfide bridges, NEM treatment would leave in vivo oxidized mCD4M61 unmodified. After lysis and incubation in the presence of 100 mM NEM, purification and TEV cleavage were performed as in the previous experiments. MALDI-TOF analysis revealed the presence of a major peak at $\mathrm{m} / \mathrm{z} 3728.14$ in perfect agreement with the calculated value for the fully reduced DRP bearing 6 NEM adducts $(m / z$ 3728.13), i.e. 3 fully reduced and alkylated disulfide bridges (Figure $8 \mathrm{~B}$ ). Analysis in the presence of NEM also revealed two other accompanying peaks at $\mathrm{m} / z 3603.06$ and 3475.96 corresponding to reduced and partially alkylated proteins bearing 5 and 4 NEM adducts, respectively. The fully oxidized peptide could not be detected in the presence of NEM

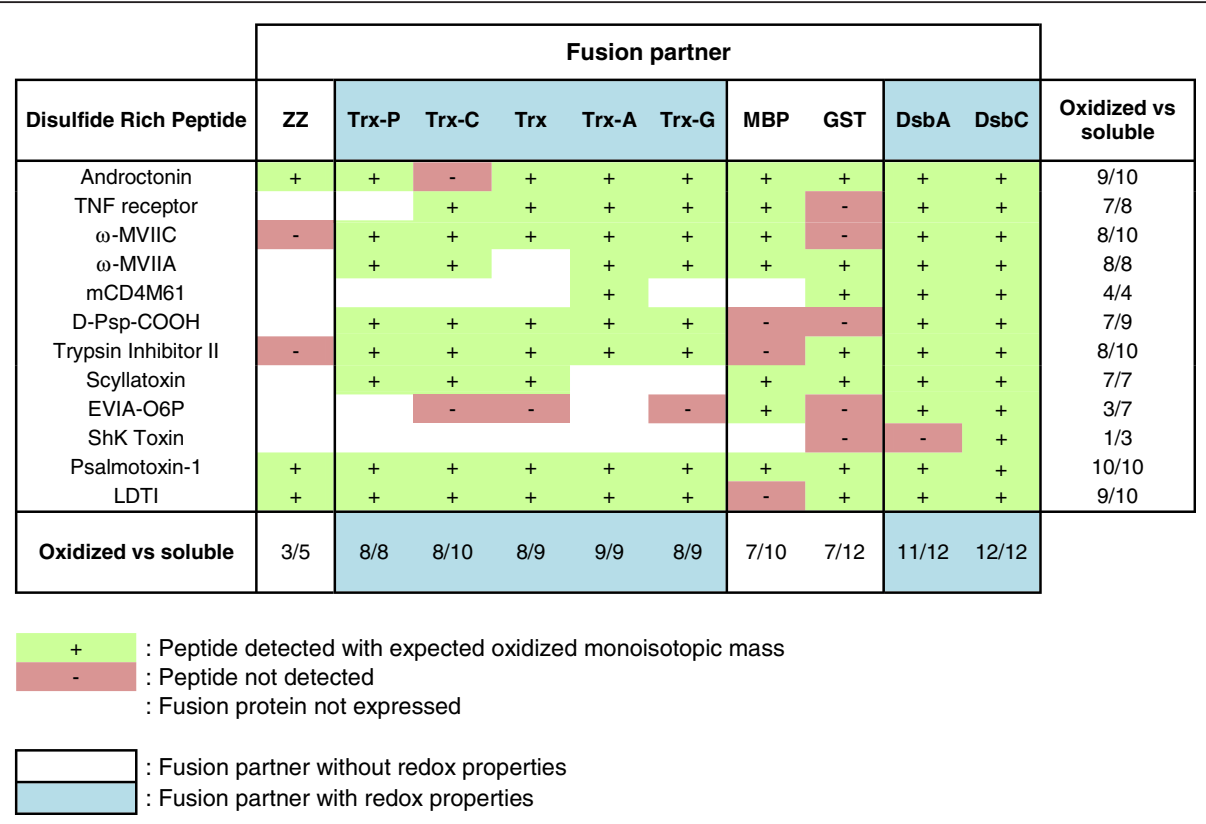

Figure 7 MALDI-TOF detection of oxidized DRPs using different fusion partners. 


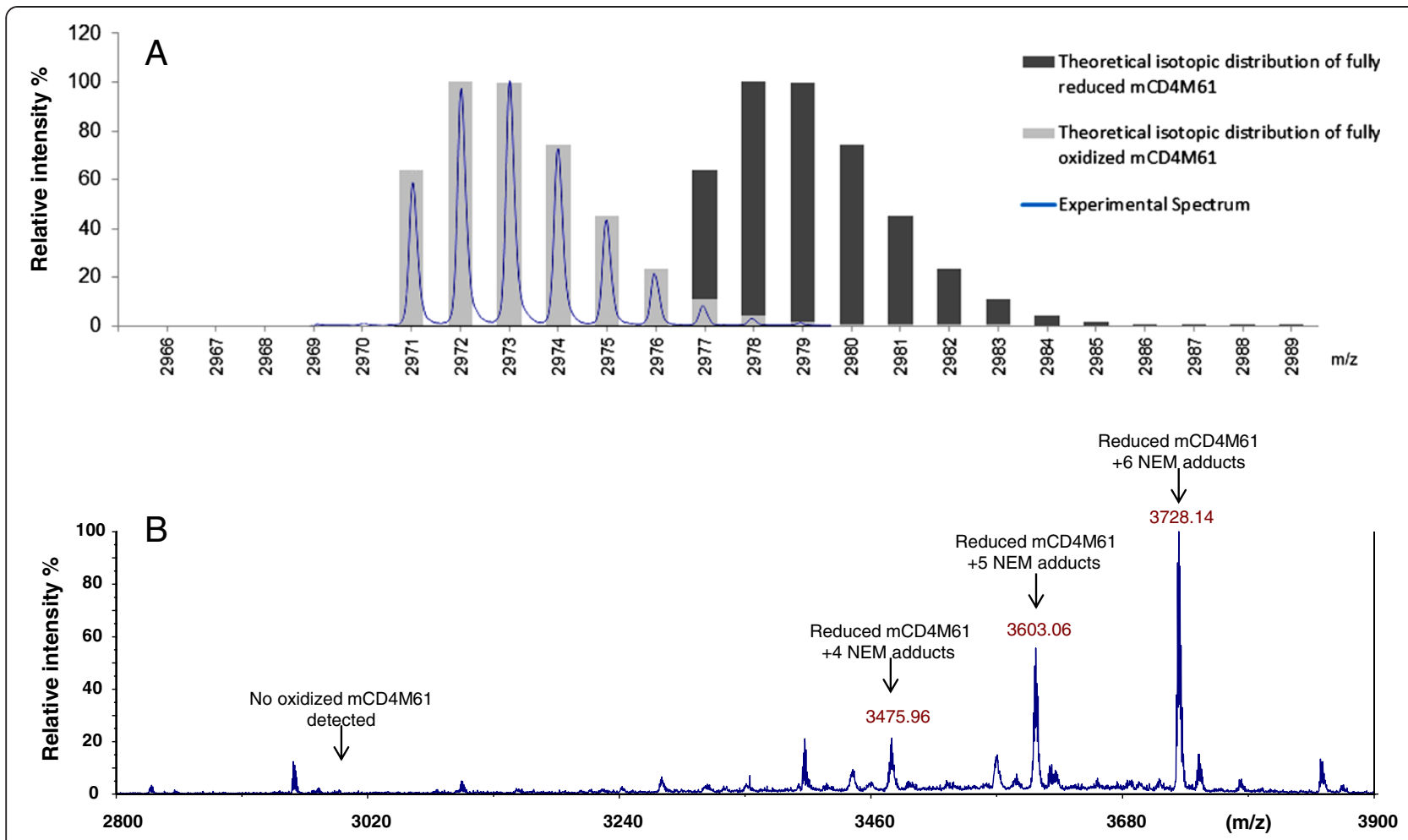

Figure 8 MALDI-TOF analysis of mCD4M61. Panel A: Comparison of the experimental MALDI-TOF spectrum obtained for the mCD4M61 with the theoretical isotopic distributions of fully reduced and fully oxidized mCD4M61. The mCD4M61 DRP was produced using DsbC as fusion partner. Panel B: Experimental MALDI-TOF spectrum obtained for the mCD4M61 using N-Ethylmaleimide as an alkylating agent during cell lysis.

suggesting that the formation of disulfide bonds in the mCD4M61 mostly occurred ex vivo (Figure 8B).

\section{Functional characterization}

Functional characterization through an activity test is one of the best methods to discriminate DRPs with their native fold and correct disulfide patterns from misfolded isoforms. Purifications from larger scale cultures using BL21 (DE3) pLysS were performed on six selected DRPs (mCD4M61, Trypsin Inhibitor II, LDTI, Thrombin Inhibitor Infestin, BPTI and MT7) for which functional tests are available. DsbC was chosen as a fusion partner because it gave the highest yields of soluble and oxidized protein in the analytical screen. Very large amounts of DsbC-DRP fusion proteins were obtained after nickel affinity purification, ranging from $90 \mathrm{mg}$ for the mCD4M61 fusion to $291 \mathrm{mg}$ for the Trypsin Inhibitor II fusion. Fusion proteins were digested with TEV protease to release the DRPs. After purification to homogeneity using reverse phase HPLC (i.e. >95\%), all DRPs were produced in quantities varying between 0.8 and $12 \mathrm{mg} / \mathrm{L}$ (Table 1), except for the muscarinic toxin MT7 for which only a few micrograms were obtained. The functional tests of these 6 proteins revealed that they were fully active (Table 1) with inhibition constants similar to the values reported in the literature [51-55], leading us to conclude that these DRPs were produced with their natively folded structures.

\section{Discussion}

We have developed a high throughput expression screen that enabled us to assess the solubility of DRPs fused to a large number of partners. We used it to study the impact of twelve fusion tags and three expression strains on the soluble level of 28 DRPs. Here, we show that a general scheme for bacterial expression in the cytoplasm of $E$. coli of genes coding for DRPs could be successfully implemented. Our approach consists of (i) cloning by recombination of genes in our set of vectors, (ii) culturing of E. coli in auto-induction media for the expression of fusion proteins in the cytoplasm (iii) lysis of bacteria, nickel affinity purification and analysis of fusion proteins, (iv) digesting with TEV and MALDI-TOF detection of oxidized DRPs. In the present study, we identified the conditions leading to soluble expression of the 28 selected DRPs in E. coli. Amongst the 1008 conditions tested in duplicates (28 proteins $\mathrm{x} 12$ fusions $\mathrm{x} 3$ strains), 196 conditions allowed soluble expression in the strain BL21 (DE3) pLysS, whereas only 44 and 50 conditions gave soluble expression for the strain Origami $\mathrm{B}$ (DE3) pLysS and SHuffle ${ }^{\odot}$ T7 Express lysY, respectively. 
Table 1 Functional validation of purified DRPs

\begin{tabular}{|c|c|c|c|c|c|c|c|c|c|}
\hline \multirow[b]{2}{*}{$\begin{array}{l}\text { Disulfide Rich } \\
\text { Peptide }\end{array}$} & \multirow[b]{2}{*}{ Fold } & \multirow[b]{2}{*}{ SS } & \multirow[b]{2}{*}{$\begin{array}{c}\text { DsbC fusion } \\
\text { produced (mg/L) }\end{array}$} & \multirow[b]{2}{*}{$\begin{array}{l}\text { Theoretical } \\
\text { DRP (mg/L) }\end{array}$} & \multirow[b]{2}{*}{$\begin{array}{l}\text { Purified DRP } \\
\text { (mg/L) }\end{array}$} & \multirow[b]{2}{*}{$\begin{array}{l}\text { DRP } \\
\text { yield }\end{array}$} & \multirow[b]{2}{*}{$\begin{array}{l}\text { Detected } \\
\text { mass }\end{array}$} & \multicolumn{2}{|c|}{ Functional test } \\
\hline & & & & & & & & Test & $\begin{array}{l}\text { Observed vs } \\
\text { Expected }\end{array}$ \\
\hline mCD4M61 & $\alpha / \beta$ & 3 & 90 & 8.9 & 3.5 & $39 \%$ & $2972.0 \pm 0.5$ & ELISA & $69 \mathrm{nM}(32 \mathrm{nM})$ \\
\hline Trypsin Inhibitor II & ICK & 3 & 291 & 30.1 & 0.9 & $3 \%$ & $3124.4 \pm 0.3$ & $\begin{array}{l}\text { Trypsin } \\
\text { Inhibition }\end{array}$ & $1.0 \mathrm{nM}(0.3 \mathrm{nM})$ \\
\hline LDTI & Kazal Type & 3 & 240 & 35.0 & 12.0 & $34 \%$ & $4572.1 \pm 0.1$ & $\begin{array}{l}\text { Trypsin } \\
\text { Inhibition }\end{array}$ & $1.8 \mathrm{nM}(1.8 \mathrm{nM})$ \\
\hline $\begin{array}{l}\text { Thrombin } \\
\text { Inhibitor Infestin }\end{array}$ & Kazal Type & 3 & 120 & 20.2 & 0.8 & $4 \%$ & $5507.7 \pm 0.2$ & $\begin{array}{l}\text { Trypsin } \\
\text { Inhibition }\end{array}$ & $1.8 \mathrm{nM}(2 \mathrm{nM})$ \\
\hline BPTI & Kunitz & 3 & 180 & 35.1 & 3.0 & $9 \%$ & $6567.6 \pm 0.1$ & $\begin{array}{l}\text { Plasmin } \\
\text { inhibition }\end{array}$ & $0.4 \mathrm{nM}(0.14 \mathrm{nM})$ \\
\hline MT7 & $3 \mathrm{FT}$ & 4 & 160 & 34.7 & 0.007 & $<0.03 \%$ & $7529.9 \pm 0.6$ & $\begin{array}{l}\text { binding } \\
\mathrm{mAChR}\end{array}$ & 5 pM (29 pM) \\
\hline
\end{tabular}

Purification from 1 liter culture of DsbC-DRP fusion in BL21 (DE3) pLysS strain.

The four-fold difference between Origami B (DE3) pLysS or SHuffle ${ }^{\oplus}$ T7 Express lysY and the BL21 (DE3) pLysS strain cannot be explained by medium composition, expression plasmids or cultivation differences, as all tests were run in the same conditions. Furthermore, thanks to the removal of the antibiotics used to maintain the $\operatorname{tr} x \mathrm{~B}^{-} /$gor genomic mutations in Origami (DE3) pLysS and SHuffle ${ }^{\odot}$ T7 Express lysY, the growth rate of the three strains were very similar. An increased lag phase was observed with the Origami B (DE3) pLysS and SHuffle ${ }^{\odot}$ T7 Express lysY but at harvesting time, in stationary phase, the optical density of the 672 cultures was in all cases around 12 (with less than $10 \%$ difference) and therefore the yield of soluble expression was considered as normalized by bacterial biomass. The low number of soluble constructs in Origami B (DE3) pLysS and SHuffle ${ }^{\oplus}$ T7 Express lysY is directly associated with low production levels. In practically all cases these two strains produced lower amounts of soluble fusion protein than BL21 (DE3) pLysS and out of the 28 DRPs, none would be favorably produced in Origami B (DE3) pLysS or SHuffle ${ }^{\oplus}$ T7 Express lysY as opposed to BL21 (DE3) pLysS. The low production level of DRPs in in Origami B (DE3) pLysS or SHuffle ${ }^{\bullet}$ T7 Express lysY was confirmed with scale-up tests (unpublished observations).

Strikingly, soluble expression of DRP fusions in Origami B (DE3) pLysS or SHuffle ${ }^{\oplus}$ T7 Express lysY are mostly observed with fusion partners lacking redox activity like GST or MBP for Origami B (DE3) pLysS and MBP for the SHuffle ${ }^{\curvearrowleft}$ T7 Express lysY. Only a few constructs were soluble using redox-active fusion partners (Trx, Trx mutants, DsbA and DsbC) and at levels below the equivalent BL21 (DE3) pLysS cultures. In spite of this, SHuffle ${ }^{\oplus}$ T7 Express lysY performed better with DsbA and DsbC than Origami B (DE3) pLysS. These results contrast with the good results of redox-active fusion partners in the strain BL21 (DE3) pLysS. This is rather unexpected since redox-active proteins such as thioredoxin or DsbC are often described as enhancing disulfide bond formation in the cytoplasm of Origami B (DE3) pLysS or equivalent strains either when coexpressed [36,56] or used as a fusion partner [57]. The low production levels in Origami B (DE3) pLysS and SHuffle ${ }^{\circledast} 7$ Express lysY strains might be linked to the fact that these strains are not as robust as other strains like BL21 (DE3) pLysS, often having altered growth parameters compared to other E. coli strains [9,58]. It is important to note that the $\operatorname{tr} x B^{-} /$gor $^{-}$strains only have the reducing pathways of the cytoplasm disrupted. This means that once a disulfide bond is formed, it is more likely to be retained than in a reducing cytoplasm, however, it does not necessarily make disulfide bond formation more efficient. Firstly, there are no added catalysts for de novo disulfide bond formation present to promote disulfide bond formation in the first place. In addition, while oxidation in the non-reducing cytoplasm can occur by the transfer of disulfide bonds to folding proteins from oxidized thioredoxin (which can no longer be reduced by the canonical pathway due to the $\operatorname{tr} x \mathrm{~B}^{-} /$gor mutation), this is relatively inefficient and slow [59]. It has been suggested that correct folding in a $\operatorname{tr} x \mathrm{~B}^{-} /$gor background is to the detriment of yield [60] and accordingly, these strains have been reported to often produce very low yields [61,62]. In fact, even with the coexpression of DsbC to catalyze disulfide isomerization, yields of oxidized correctly folded protein can only be improved if (i) the expressed protein is soluble (perhaps necessitating the use of additional solubilizing fusion tags), and (ii) if the disulfide bonds are already formed (either spontaneously or by interaction with other disulfide bond donor proteins, including DsbC itself). These reasons may explain why soluble yields from SHuffle strains have not been improved beyond those found in Origami strains for the expression of certain proteins 
[58]. It must be noted however, that use of Origami B (DE3) pLysS or SHuffle ${ }^{\oplus}$ T7 Express lysY might necessitate screening of additional parameters such as medium, induction conditions, temperatures [36] or protein coexpression that were not investigated in our study. However, in our set-up, the difference observed between BL21 (DE3) pLysS and Origami B (DE3) pLysS or SHuffle ${ }^{\bullet}$ T7 Express lysY was significant and we have chosen to focus only on the strain BL21 (DE3) pLysS.

In contrast to Origami B (DE3) pLysS or SHuffle ${ }^{\oplus} \mathrm{T} 7$ Express lysY, the success rates of DRPs expressed as fusions in the cytoplasm of BL21 (DE3) pLysS was high $(196 / 336)$. For every DRP of interest, multiple soluble expression conditions were observed. Our procedure yields relatively high quantities of fusion proteins after nickel affinity purification: approximately $20 \%$ of the conditions tested led to estimated quantities greater than 10 milligrams per liter of culture (65/336). Quantities of fusion proteins are higher with MBP, GST, DsbA and DsbC than for the other fusion partners. Use of thioredoxin and thioredoxin mutants could be an interesting option, because of their lower molecular weight compared to the other fusion partners (15 kDa vs $25-40$ $\mathrm{kDa}$ ), thereby providing a greater proportional yield of DRPs to fusion tag [13]. Even so, from the data collected here, the impact of the thioredoxin mutations is difficult to appreciate, both in terms of solubilization and quantities of fusion.

To investigate the redox states of the DRPs when produced in fusion, we have coupled the solubility screen with MS detection of the proteins whenever possible. MALDI-TOF analyses revealed that detectable amounts of oxidized proteins can be cleaved off from the fusion partners in a very high number of cases using BL21 (DE3) pLysS as a production strain. These observations raised the question of whether the disulfide bond formation occurred in vivo or ex vivo. The ex vivo formation of disulfide bonds is a hypothesis also suggested previously by Tedford et al. [63] based on the equivalent oxidized production of a single spider toxin in BL21 versus a trx strain. Thus, to test this hypothesis, the disulfide bond status of mCD4M61 fused with DsbC was examined by subjecting the lysate to addition of $\mathrm{N}$ ethylmaleimide at the moment of cell lysis. In contrast with the untreated samples, oxidized mCD4M61 could not be detected after purification and TEV cleavage, indicating that the disulfide bond formation probably occurred ex vivo. The strain BL21 (DE3) pLysS allows the production of high amounts of DRPs fused with different partners but its cytoplasm probably remains an unfavorable environment for the formation of disulfide bonds. In contrast, the purification steps after cell lysis probably provide a more favorable environment for the DRP to reach an oxidized state. Purification steps not only remove the reducing pathway components that could hamper the disulfide bond formation but are also performed at $\mathrm{pH} 8$, rendering protein thiols (typically $\left.\mathrm{p} K_{\mathrm{a}} \sim 8-9\right)$ very reactive. The buffer also has higher concentrations of molecular oxygen that can act as final electron acceptors and thus be the driving force leading to the oxidation of the peptide.

The data collected suggest that the redox properties of the fusion partner might have an effect on the folding of the DRPs. First, we have shown that either redoxinactive (MBP, GST) or redox-active (DsbC, DsbA) fusion partners are able to produce significant quantities of fusion proteins for many DRPs. However, MALDITOF experiments revealed that constructs for which oxidized peptide could not be detected are, in most cases, fusions without redox properties (e.g. MBP, GST). In contrast, most oxidized DRPs are detected when thioredoxins, DsbA or DsbC were used as fusion partners. These observations support the hypothesis that redox activity of the carrier has an influence on the folding of the DRP, even if oxidation occurs ex vivo. When using fusion partners with redox activity, the partner could not only improve the solubility of the folding intermediates but could also assist the DRPs to reach their native oxidized form. To further confirm this hypothesis, it could be interesting to investigate the effect of inactive redox variants of those partners on the yield of active DRP.

Under our criterions, use of DsbC as a fusion partner in the strain BL21 (DE3) pLysS was the most potent combination tested. Indeed, use of DsbC yielded soluble fusions for almost all the DRPs $(27 / 28)$ but also has the strongest effect on the quantity of fusions produced. When DsbC is used as a fusion partner, more than $67 \%$ of the constructs $(18 / 28)$ are produced with yields exceeding $10 \mathrm{mg} / \mathrm{L}$ at the screening scale. The true magnitude of those yields becomes even more apparent when proteins are expressed at larger scale (1 liter) with up to $290 \mathrm{mg} / \mathrm{L}$ for the DsbC fusions. Foremost, mass spectrometry experiments also revealed that DsbC generates oxidized folded DRPs in a significant number of cases. The favorable results obtained with DsbC can be explained by its excellent solubilization potential but most importantly by its isomerase and chaperonin activities [17], which are a considerable advantage to assist DRPs to reach their native active state. Most importantly, depending on the DRP properties, the proportion of folded DRP can be very significant as attested by the good yields obtained for scale-up production and purification using DsbC as fusion partner (e.g. LDTI, BPTI, mCD4M61, Table 1).

Only a few articles available in the literature report the successful production of oxidized DRPs in the $E$. coli cytoplasm without the use of $\operatorname{trx} \mathrm{B}^{-} /$gor $^{-}$strains or 
in vitro refolding steps. Bogomolovas et al. reported the production of active viscotoxin A3 containing three disulfide bonds using thioredoxin as a fusion partner [13]. Similarly, Mac et al. reported production of Endothelin1 containing two disulfides using thioredoxin [64], while Tedford et al. used a GST-fusion for the production of an insecticidal spider toxin containing three disulfide bonds [63]. Our present work shows that many other DRPs can be produced and purified to homogeneity at the milligram scale using our protocols, spanning a diversity of folds $(\alpha / \beta$, ICK, Kazal type, Kunitz). We observed that some proteins tested were more prone to adopt their oxidized native conformation than others. As an illustration, we can see that mCD4M61, Psalmotoxin1 and LDTI were detected in MALDI-TOF analyses in virtually all cases, independently of the fusion partner used. On the contrary, EVIA and ShK toxins were only detected in a few conditions tested. In addition, yields from large-scale expression of DRPs were quite casedependent, ranging from a few micrograms for the hardto-express muscarinic toxin MT7 containing four disulfide bonds to more than $12 \mathrm{mg}$ for LDTI. In some cases, the amounts of DRPs obtained after all production and purification steps are lower than what could be expected from the amounts of fusion protein produced. It has to be noted that no individual optimization was performed to increase yield for individual DRPs. Thus, one could reasonably expect to increase yields by improving the process for a specific protein (buffers, purification, choice of the protease) or by performing further in vitro refolding steps.

While this optimization would be an interesting option to increase yields, one should also consider other tools to encourage increased efficiency of correct peptide folding. Indeed, the ratios between purified DRPs versus purified fusion proteins suggest that the folding of some proteins is particularly incomplete (e.g. MT7, Thrombin Inhibitor Infestin or Trypsin Inhibitor II). Among the further improvements envisioned, it may be of interest to express concomitantly and in stoichiometric excess a second redox-active protein (oxidase or isomerase) to boost the positive effect of DsbC. Besides that, the lysis buffer components could possibly have an influence on the DRP folding. Given the hypothesis of an ex vivo oxidation it would be of interest to investigate the effect of introducing oxidized/reduced glutathione which are classically used for in vitro DRP refolding strategies. Additionally, use of tools promoting the in vivo folding and oxidation of the DRP would be an interesting option to achieve higher productivity. Co-expression of sulfhydryl oxidases $[61,62,65]$ or addition of redox molecules in the medium [66] could be promising options to promote the formation of disulfides bonds in vivo. These tools could be additionally introduced in our screen without significant modification of the process.

\section{Conclusion}

Our high throughput screening approach allows the systematic investigation of the solubilizing and folding influence of various partners in the strains BL21 (DE3) pLysS, Origami B (DE3) pLysS and SHuffle ${ }^{\oplus}$ T7 Express lysY for the production of soluble DRPs. In spite of its reducing cytoplasm, BL21 (DE3) pLysS is a very efficient strain for the production of DRPs in fusion with solubilizing partners. In our set-up, $\operatorname{tr} x \mathrm{~B}^{-} /$gor $^{-}$strains Origami B (DE3) pLysS and SHuffle ${ }^{\bullet}$ T7 Express lysY yielded only very low amounts of fusion proteins. Many DRPs are found oxidized after production in BL21 (DE3) pLysS, most probably because of post-lytic oxidation reactions. In many ways, use of DsbC as a fusion partner in the strain BL21 (DE3) pLysS was the most potent combination tested. Our protocols allow the production of a large diversity of DRPs using DsbC as a fusion partner, leading to pure active DRPs at milligram scale in many cases. Thus, this work should facilitate the study of DRPs with therapeutic or biotechnological interest whose production was previously a limiting step.

\section{Materials and methods}

Design and construction of the expression plasmid library Synthetic genes optimized for recombinant expression of miniproteins in E. coli were ordered from Geneart AG. These genes contain the sequence coding for a TEV protease cleavage site (ENLYFQ/G) followed by the sequence corresponding to the DRP (see Additional file 1: Table S1), with Gateway recombination sites on each extremity of the gene. These synthetic constructs were cloned by Gateway ${ }^{\text {max }}$ BP cloning technology using pDONR221 as a donor vector. Twelve Gateway destination vectors were used in this study. Each contains one of the eleven fusion partners and a 6HIS tag for protein purification located either on the $\mathrm{N}$ - or C-terminal side of the fusion partner and the twelfth vector containing 6HIS alone (see Additional file 2: Table S2). The 28 entry clones were in turn recombined using the Gateway ${ }^{\text {tw }}$ LR cloning technology using one of the 12 Gateway destination vectors. Thus, a total of 336 different expression plasmids were created. All cloning steps (Gateway LR cloning of the 28 pENTR clones in 12 destination vectors, DNA purifications, bacterial transformations and cultures) were accomplished within a week using high throughput compliant protocols detailed elsewhere [9].

\section{High throughput protein expression screening}

In this study, all cultures were grown in auto-induction medium ZYP-5052 supplemented with antibiotics both for small-scale expression screens (in BL21 (DE3) pLysS, Origami (DE3) pLysS and SHuffle ${ }^{\circ}$ T7 Express lysY) or scale-up expression (in BL21 (DE3) pLysS). ZYP-5052 medium is a buffered complex medium containing 
glucose, lactose and glycerol formulated to induce protein expression after glucose depletion [50]. Expressions were performed using a standardized two-step process. In the first part of fermentation, cells were grown at $37^{\circ}$ $\mathrm{C}$ to quickly reach the glucose depletion phase just before the induction. After that step (4 hours) the temperature was lowered to $17^{\circ} \mathrm{C}$ for $18 \mathrm{~h}$ to favor protein folding and soluble protein expression.

All steps were carried out in 24 or 96 deep-well plates (DW24 and DW96, respectively). Expression strains were obtained after a heat-shock transformation of competent cells with the expression plasmids. Transformed cells were used to inoculate pre-cultures in DW96 plates containing $1 \mathrm{~mL}$ of LB media in each well. The following morning, $100 \mu \mathrm{L}$ of the pre-culture broth was used to inoculate $4 \mathrm{~mL}$ of ZYP-5052 medium. Cultivation was carried out using DW24 plates to increase the biomass compared to DW96 cultures. After an overnight incubation at $17^{\circ} \mathrm{C}$, cells were pelleted by centrifugation, resuspended in lysis buffer and transferred into DW96 and frozen at $-80^{\circ} \mathrm{C}$. After thawing the cells the lysate was purified using an automated nickel affinity procedure as described in Figure 2. The whole procedure for the BL21 (DE3) pLysS and Origami B (DE3) pLysS (672 cultures followed by purification and analysis), was performed within a week and reproduced a second time to confirm the results (these protocols have been detailed elsewhere $[9,49]$ ) while the SHuffle ${ }^{\circ}$ T7 Express lysY experiment was done on a separate week.

\section{Identification of soluble expression conditions}

In this study, the analysis of the purified protein yields (as well as the efficiency of TEV cleavage) was performed on a Labchip GXII (Caliper, USA) microfluidic high throughput system, which was more adapted to the throughput of this work than traditional SDS-PAGE analysis. This analysis (done following the manufacturer's instructions) provides an estimation of the molecular weight, concentration and purity of the proteins with a detection limit of approximately $0.1 \mathrm{mg} / \mathrm{L}$ of culture. Proteins below $5 \mathrm{kDa}$ could not be assessed by this method due to system limits. For the DRPs below this molecular weight, the cleavage efficiency was initially assessed by the disappearance of the fusion-DRP species. The molecular weight of the free DRP was only confirmed by mass spectrometry at scale up.

\section{Large-scale cultures, purification and cleavage of DsbC-DRPs}

For larger scale production of recombinant proteins, a Fernbach flask containing $1 \mathrm{~L}$ of ZYP-5052 auto-induction medium and the appropriate antibiotics was inoculated with an overnight culture of BL21 (DE3) pLysS to reach 0.05 O.D. at $600 \mathrm{~nm}$. Cultures were performed using the two-step protocol $\left(4 \mathrm{~h}\right.$ at $37^{\circ} \mathrm{C}$ then $18 \mathrm{~h}$ at $17^{\circ} \mathrm{C}$ ). At the end of culture, cells were then harvested by centrifugation $\left(4500 \times g, 30 \mathrm{~min}, 4^{\circ} \mathrm{C}\right)$ and the pellet was resuspended in $50 \mathrm{~mL}$ of lysis buffer (100 mM Tris-HCl, pH 8, $150 \mathrm{mM}$ $\mathrm{NaCl}, 5 \%$ glycerol). Lysis of cells was performed using a cell disrupter (Constant System Ltd). The lysate was cleared by centrifugation $\left(18000 \mathrm{rpm}, 30 \mathrm{~min}, 4^{\circ} \mathrm{C}\right)$ and the supernatant loaded onto a $5 \mathrm{~mL}$ HisTrap FF column (GE-Healthcare Bio-Sciences). The 6HIStagged fusion proteins were then eluted with a linear gradient ( 0 to $100 \% \mathrm{~B}$ in $30 \mathrm{~min}$ at a flow rate of $2 \mathrm{~mL} /$ min) of buffer B (100 mM Tris- $\mathrm{HCl}, \mathrm{pH} 8,150 \mathrm{mM}$ $\mathrm{NaCl}, 5 \%$ glycerol, $500 \mathrm{mM}$ imidazole) in buffer A (100 $\mathrm{mM}$ Tris- $\mathrm{HCl}, \mathrm{pH} 8,150 \mathrm{mM} \mathrm{NaCl}, 5 \%$ glycerol, 40 $\mathrm{mM}$ imidazole). The fractions containing the 6HIStagged fusion protein were pooled and dialyzed for $3 \mathrm{~h}$ against lysis buffer using a Spectra/Por ${ }^{\circ}$ Dialysis Membrane (MWCO: 3500). The protein of interest was then cleaved with $10 \%(\mathrm{w} / \mathrm{w}) \mathrm{TEV}$ protease overnight at $4{ }^{\circ} \mathrm{C}$ and purified by RP-HPLC. RP-HPLC purification was performed using a semi-preparative C4 column (Vydac 214TP1010, $10 \mu \mathrm{m}, 300 \AA$, $10 \times 250 \mathrm{~mm}$ ) using a linear gradient $0-60 \%$ in 30 min of solvent $\mathrm{B}(100 \%$ acetonitrile, $0.09 \%$ TFA $)$ in solvent $\mathrm{A}\left(100 \% \mathrm{H}_{2} \mathrm{O}, 0.1 \%\right.$ TFA) with a flow rate of $4 \mathrm{~mL} / \mathrm{min}$. After HPLC purification, DRPs were lyophilized and solubilized in the appropriate buffer for further studies.

\section{Characterization by mass spectrometry}

Detection of oxidized DRPs used MALDI-TOF and LTQOrbitrap. The purified fusion protein samples were digested by TEV protease at $4^{\circ} \mathrm{C}$. Samples were loaded onto $\mathrm{C} 18$ reverse phase ZipTips, desalted and eluted by $70 \%$ acetonitrile/ $\mathrm{H}_{2} \mathrm{O} / 0.1 \%$ TFA before spotting on a MALDI plate with 4-CHCA matrix at $10 \mathrm{mg} / \mathrm{mL}$. MALDITOF analyses were performed on a MALDI-TOF/TOF ${ }^{\text {six }}$ 4800 Analyzer from AB-SCIEX (Foster City, CA). The isotopic pattern measured was compared with the theoretical one determined from the amino acid sequences using DataExplorer software (Version 4.9, Applied Biosystems).

High resolution/high mass accuracy measurements were performed on an LTQ-Orbitrap instrument (Thermo, San Jose, CA) by UHPLC-MS essentially as described previously [67]. Briefly, DRP samples were loaded and separated on a C18 Hypersil GOLD column (2.1 mm x $150 \mathrm{~mm}, 175 \AA, 1.9 \mu \mathrm{m}$, ThermoScientific) at a flow rate of $300 \mu \mathrm{L} / \mathrm{min}$ with a linear gradient of 0 to $80 \% \mathrm{~B}$ in $10 \mathrm{~min}$ (with solvent $\mathrm{A}: \mathrm{H}_{2} \mathrm{O}$ containing $0.1 \%$ formic acid and solvent $\mathrm{B}$ : acetonitrile containing $0.1 \%$ formic acid). MS acquisition was performed in the positive ion mode from $\mathrm{m} / z 500$ to 2000 using a resolution set at $30000($ at $\mathrm{m} / \mathrm{z}$ 400). The resulting mass spectra were deconvoluted using ProMass software (ThermoScientific). 


\section{Functional characterization of purified DRPs}

Enzyme inhibition by the inhibitors was tested in competition experiments. For plasmin tests, we used $6 \mu \mathrm{M}$ fluorogenic substrate N-succinimyl-Ala-Phe-Lys-AMC (Sigma Aldrich) and plasmin (Sigma Aldrich) at $0.1 \mathrm{nM}$ concentration in $100 \mu \mathrm{L}$ of PBS buffer. For Trypsin tests, we used $1.8 \mu \mathrm{M}$ fluorogenic substrate Mca-R-P-K-P-VE-NVal-W-R-P-K(Dnp)-NH2 (R\&D Systems) and Trypsin (Sigma Aldrich) at $0.4 \mathrm{nM}$ concentration in $200 \mu \mathrm{L}$ of 50 $\mathrm{mM}$ Tris- $\mathrm{HCl}$ buffer $\mathrm{pH}$ 6.8, $100 \mathrm{mM} \mathrm{NaCl}, 10 \mathrm{mM}$ $\mathrm{CaCl}_{2}$.

The substrate and enzyme concentrations for the experiments were chosen so as to remain well below $10 \%$ of substrate utilization and to observe the initial rates. For each inhibitor, the percentage of inhibition was determined in duplicate experiments at four inhibitor concentrations, chosen to observe a $20-80 \%$ range of inhibition. After $1 \mathrm{~h}$ incubation with shaking at $25^{\circ} \mathrm{C}$, inhibition assays were performed by recording the fluorescence increase induced by the cleavage of fluorogenic substrate, using 96-well nonbinding surface plates (3650 Corning-Costar plates). Fluorescence signals were monitored using a Fluoroscan Ascent photon counter spectrophotometer (ThermolabSystems) equipped with a temperature control device and a plate shaker. $\mathrm{K}_{\mathrm{i}}$ values were determined using the method proposed by Horovitz and Levitski [68].

The property of miniCD4 (mCD4M61) to inhibit the binding of HIV-envelope was evaluated by in vitro competitive ELISA as previously described [3]. The effect of MT7 on the equilibrium bonding of [3H]NMS on M1 muscarinic receptor was determined in inhibition experiments as previously described [51].

\section{Additional files}

Additional file 1: Table S1. Disulfide-rich proteins and their characteristics. Sequences beginning with SSCS have a modified Nterminus sequence, however the cysteine position and pattern has been conserved.

Additional file 2: Table S2. Destination plasmids used in this study.

Additional file 3: Table S3. Molecular masses of the 25 DsbC-DRP fusions as determined by LTQ-Orbitrap mass spectrometer.

\begin{abstract}
Abbreviations
BPTI: Bovine pancreatic trypsin inhibitor; LDTI: Leech-Derived Tryptase Inhibitor; DRP: Disulfide-rich proteins; ICK: Inhibitor Cystine Knot; 3FT: Threefinger toxin; TDPI: Tick-derived Protease Inhibitor; RBI: Ragi bifunctional inhibitor; MALDI-TOF: Matrix-assisted laser desorption/ionization-Time of flight.
\end{abstract}

\section{Competing interests}

The authors declare that they have no competing interests.

\section{Authors' contributions}

$H N, R V, D S, M G, P C$ and VD have set up and designed the study; HN and MM cloned the destination plasmids; RV designed and supervised the high throughput experiments; HN, RV, NS have cloned the expression plasmids and performed high throughput screening recombinant expression experiments; HN, CFG, OHPR, FB and BD have performed scale-up expression, purification and characterization of selected DRPs. HN, FF and RT have performed mass spectroscopy experiments; HN, RV and VD have analyzed the data and written the manuscript; all authors discussed the results and commented on the manuscript and all authors read and approved the final manuscript.

\section{Acknowledgements}

The authors would like to thank Dr P. Belin, Dr L. Martin, Dr P. Kessler for their valuable comments and suggestions. The authors also wish to acknowledge Dr F. Biteau and Pr. H. Darbon for critical reviewing of the manuscript. They are also grateful to Mr Steven Dubois and Mr Olivier Combes for their technical support.

\section{Funding}

This work was supported by the ANR PFTV, the GIS IBiSA, the ANR FRISBI (10-INSB-05-01) and by INSTRUCT (ESFRI). Dr Natalie Saez was supported by the VENOMICS project, European project grant № 278346 (FP7 HEALTH 20112015).

\section{Author details}

${ }^{1}$ CEA, iBiTec-S, Service d'Ingénierie Moléculaire des Protéines (SIMOPRO), CEA Saclay, Gif sur Yvette F-91191, France. ${ }^{2}$ Architecture et Fonction des Macromolécules Biologiques (A.F.M.B.), UMR7257 CNRS, Université AixMarseille, Case 932, 163 Avenue de Luminy, Marseille cedex 9 13288, France. ${ }^{3}$ CEA, iBiTec-S, Service de Pharmacologie et d'Immunoanalyse (SPI), CEA Saclay, Gif sur Yvette F-91191, France.

Received: 23 January 2013 Accepted: 28 March 2013 Published: 22 April 2013

\section{References}

1. King GF: Venoms as a platform for human drugs: translating toxins into therapeutics. Expert Opin Biol Ther 2011, 11:1469-1484.

2. Miljanich GP: Ziconotide: Neuronal calcium channel blocker for treating severe chronic pain. Curr Med Chem 2004, 11:3029-3040.

3. Martin L, Stricher F, Misse D, Sironi F, Pugniere M, Barthe P, Prado-Gotor R, Freulon I, Magne X, Roumestand C, et al: Rational design of a CD4 mimic that inhibits HIV-1 entry and exposes cryptic neutralization epitopes. Nat Biotechnol 2003, 21:71-76.

4. Smith GP, Patel SU, Windass JD, Thornton JM, Winter G, Griffiths AD: Small binding proteins selected from a combinatorial repertoire of knottins displayed on phage. J Mol Biol 1998, 277:317-332.

5. Hancock REW, Sahl HG: Antimicrobial and host-defense peptides as new anti-infective therapeutic strategies. Nat Biotechnol 2006, 24:1551-1557.

6. Kimura RH, Cheng Z, Gambhir SS, Cochran JR: Engineered knottin peptides: a New class of agents for imaging integrin expression in living subjects. Cancer Res 2009, 69:2435-2442.

7. Demain AL, Vaishnav P: Production of recombinant proteins by microbes and higher organisms. Biotechnol Adv 2009, 27:297-306.

8. Braud S, Moutiez M, Belin P, Abello N, Drevet P, Zinn-Justin S, Courcon M, Masson C, Dassa J, Charbonnier JB, et al: Dual expression system suitable for high-throughput fluorescence-based screening and production of soluble proteins. J Proteome Res 2005, 4:2137-2147.

9. Vincentelli R, Cimino A, Geerlof A, Kubo A, Satou Y, Cambillau C: Highthroughput protein expression screening and purification in Escherichia coli. Methods 2011, 55:65-72.

10. Groisillier A, Herve C, Jeudy A, Rebuffet E, Pluchon PF, Chevolot Y, Flament D, Geslin C, Morgado IM, Power D, et al: MARINE-EXPRESS: taking advantage of high throughput cloning and expression strategies for the post-genomic analysis of marine organisms. Microb Cell Fact 2010, 9:45.

11. Xiao R, Anderson S, Aramini J, Belote R, Buchwald WA, Ciccosanti C, Conover K, Everett JK, Hamilton K, Huang YJ, et al: The high-throughput protein sample production platform of the Northeast Structural Genomics Consortium. J Struct Biol 2010, 172:21-33.

12. Douzi BGA, Gilles N, Darbon H, Marchot P, Vincentelli R, Barbier JBE, Marchot $P$, Mattei $C$, Servent $D$ : A new system for expressing recombinant animal toxins in E. coli. In Advances and new technologies in toxinology. France: Collection Rencontres en Toxinologie, Publications de la SFET, ChâtenayMalabry; 2010:149-152. 
13. Bogomolovas J, Simon B, Sattler M, Stier G: Screening of fusion partners for high yield expression and purification of bioactive viscotoxins. Protein Expr Purif 2009, 64:16-23.

14. Chen YQ, Zhang SQ, Li BC, Qiu W, Jiao B, Zhang J, Diao ZY: Expression of a cytotoxic cationic antibacterial peptide in Escherichia coli using two fusion partners. Protein Expr Purif 2008, 57:303-311.

15. Torres FS, Silva CN, Lanza LF, Santos AV, Pimenta AM, De Lima ME, Diniz MR: Functional expression of a recombinant toxin - rPnTx2-6 - active in erectile function in rat. Toxicon 2010, 56:1172-1180.

16. Lyukmanova EN, Shulepko MA, Shenkarev ZO, Dolgikh DA, Kirpichnikov MP: In vitro production of three-finger neurotoxins from snake venoms, a disulfide rich proteins. Problems and their solutions (Review). Russ $J$ Bioorg Chem 2010, 36:137-145.

17. de Marco A: Strategies for successful recombinant expression of disulfide bond-dependent proteins in Escherichia coli. Microb Cell Fact 2009, 8:26.

18. Weissman JS, Kim PS: Reexamination of the folding of BPTI: predominance of native intermediates. Science 1991, 253:1386-1393.

19. Creighton TE: The disulfide folding pathway of BPTI. Science 1992, 256:111-114.

20. Chang JY: The disulfide folding pathway of tick anticoagulant peptide (TAP), a Kunitz-type inhibitor structurally homologous to BPTI. Biochemistry 1996, 35:11702-11709.

21. Bronsoms S, Pantoja-Uceda D, Gabrijelcic-Geiger D, Sanglas L, Aviles FX, Santoro J, Sommerhoff CP, Arolas JL: Oxidative folding and structural analyses of a kunitz-related inhibitor and its disulfide intermediates: functional implications. J Mol Biol 2011, 414:427-441.

22. de Marco A, Vigh L, Diamant S, Goloubinoff P: Native folding of aggregation-prone recombinant proteins in Escherichia coli by osmolytes, plasmid- or benzyl alcohol-overexpressed molecular chaperones. Cell Stress Chaperones 2005, 10:329-339.

23. Baneyx F, Mujacic M: Recombinant protein folding and misfolding in Escherichia coli. Nat Biotechnol 2004, 22:1399-1408.

24. Saez NJ, Mobli M, Bieri M, Chassagnon IR, Malde AK, Gamsjaeger R, Mark AE, Gooley PR, Rash LD, King GF: A dynamic pharmacophore drives the interaction between Psalmotoxin-1 and the putative drug target acidsensing ion channel 1a. Mol Pharmacol 2011, 80:796-808.

25. Anangi R, Rash LD, Mobli M, King GF: Functional expression in escherichia coli of the disulfide-rich sea anemone peptide APETx2, a potent blocker of acid-sensing lon channel 3. Mar Drugs 2012, 10:1605-1618.

26. Heras B, Kurz M, Shouldice SR, Martin JL: The name's bond......disulfide bond. Curr Opin Struct Biol 2007, 17:691-698.

27. Ito K, Inaba K: The disulfide bond formation (Dsb) system. Curr Opin Struct Biol 2008, 18:450-458.

28. Fernandez C, Salinas G, Pellizza L, Margenat M, Flo M: Tuned Escherichia coli as a host for the expression of disulfide-rich proteins. Biotechnol $J$ 2011, 6:686-699

29. Yoon SH, Kim SK, Kim JF: Secretory production of recombinant proteins in escherichia coli. Recent Pat Biotechnol 2010, 4:23-29.

30. Bessette PH, Aslund F, Beckwith J, Georgiou G: Efficient folding of proteins with multiple disulfide bonds in the Escherichia coli cytoplasm. Proc Natl Acad Sci USA 1999, 96:13703-13708.

31. Derman Al, Prinz WA, Belin D, Beckwith J: Mutations that allow disulfide bond formation in the cytoplasm of Escherichia-coli. Science 1993 262:1744-1747.

32. Xu Y, Yasin A, Tang R, Scharer JM, Moo-Young M, Chou CP: Heterologous expression of lipase in Escherichia coli is limited by folding and disulfide bond formation. Appl Microbiol Biotechnol 2008, 81:79-87.

33. Saejung W, Puttikhunt C, Prommool T, Sojikul P, Tanaka R, Fujiyama K, Malasit P, Seki T: Enhancement of recombinant soluble dengue virus 2 envelope domain III protein production in Escherichia coli trxB and gor double mutant. J Biosci Bioeng 2006, 102:333-339.

34. Faulkner MJ, Veeravalli K, Gon S, Georgiou G, Beckwith J: Functional plasticity of a peroxidase allows evolution of diverse disulfide-reducing pathways. Proc Natl Acad Sci USA 2008, 105:6735-6740.

35. Skretas G, Carroll S, DeFrees S, Schwartz MF, Johnson KF, Georgiou G: Expression of active human sialyltransferase ST6GaINAcl in Escherichia coli. Microb Cell Fact 2009, 8:50.

36. Lobstein J, Emrich CA, Jeans C, Faulkner M, Riggs P, Berkmen M: SHuffle, a novel Escherichia coli protein expression strain capable of correctly folding disulfide bonded proteins in its cytoplasm. Microb Cell Fact 2012, $11: 56$
37. Jonathan Beckwith FA, Paul H, Bessette George G, Daniel R, Lim JEa, United states: President and Fellows of Harvard College, Cambridge, MA (US; Board of Regents: Compositions and methods for production of disulfide bond containing proteins in host cells. Austin, TX (US): University of Texas System; 2008.

38. Sorensen HP, Mortensen KK: Soluble expression of recombinant proteins in the cytoplasm of Escherichia coli. Microb Cell Fact 2005, 4:1.

39. Esposito D, Chatterjee DK: Enhancement of soluble protein expression through the use of fusion tags. Curr Opin Biotechnol 2006, 17:353-358.

40. Walls D, Loughran ST: Tagging recombinant proteins to enhance solubility and aid purification. Methods Mol Biol 2011, 681:151-175.

41. Smith DB, Johnson KS: Single-step purification of polypeptides expressed in escherichia-coli as fusions with glutathione S-transferase. Gene 1988, 67:31-40.

42. Maina CV, Riggs PD, Grandea AG 3rd, Slatko BE, Moran LS, Tagliamonte JA, McReynolds LA, Guan CD: An Escherichia coli vector to express and purify foreign proteins by fusion to and separation from maltose-binding protein. Gene 1988, 74:365-373.

43. Drevet $P$, Lemaire C, Gasparini S, ZinnJustin S, Lajeunesse E, Ducancel F, Pinkasfeld S, Courcon M, Tremeau O, Boulain JC, Menez A: High-level production and isotope labeling of snake neurotoxins, disulfide-rich proteins. Protein Expr Purif 1997, 10:293-300.

44. Huth JR, Bewley CA, Jackson BM, Hinnebusch AG, Clore GM, Gronenborn AM: Design of an expression system for detecting folded protein domains and mapping macromolecular interactions by NMR. Protein Sci 1997, 6:2359-2364

45. Dyson MR, Shadbolt SP, Vincent KJ, Perera RL, McCafferty J: Production of soluble mammalian proteins in Escherichia coli: identification of protein features that correlate with successful expression. BMC Biotechnol 2004, 4:32.

46. Jurado P, Ritz D, Beckwith J, de Lorenzo V, Fernandez LA: Production of functional single-chain Fv antibodies in the cytoplasm of Escherichia coli. J Mol Biol 2002, 320:1-10.

47. Gleiter S, Bardwell JC: Disulfide bond isomerization in prokaryotes. Biochim Biophys Acta 2008, 1783:530-534.

48. Marsischky G, LaBaer J: Many paths to many clones: a comparative look at high-throughput cloning methods. Genome Res 2004, 14:2020-2028.

49. Saez NJ, Vincentelli R: High-throughput expression screening and purification of recombinant proteins in E. coli. Methods Mol Biol 2013, in press.

50. Studier FW: Protein production by auto-induction in high density shaking cultures. Protein Expr Purif 2005, 41:207-234.

51. Fruchart-Gaillard C, Mourier G, Marquer C, Ménez A, Servent D: Identification of various allosteric interaction sites on M1 muscarinic receptor using 125I-Met35-oxidized muscarinic toxin 7. Mol Pharmacol 2006, 69:1641-1651.

52. Campos ITN, Amino R, Sampaio CAM, Auerswald EA, Friedrich T, Lemaire HG, Schenkman S, Tanaka AS: Infestin, a thrombin inhibitor presents in Triatoma infestans midgut, a Chagas' disease vector: gene cloning, expression and characterization of the inhibitor. Insect Biochem Mol Biol 2002, 32:991-997.

53. Heitz A, Avrutina O, Le-Nguyen D, Diederichsen U, Hernandez JF, Gracy J, Kolmar $\mathrm{H}$, Chiche L: Knottin cyclization: impact on structure and dynamics. BMC Struct Biol 2008, 8:54.

54. Stubbs MT, Morenweiser R, Sturzebecher J, Bauer M, Bode W, Huber R, Piechottka GP, Matschiner G, Sommerhoff CP, Fritz H, Auerswald EA: The three-dimensional structure of recombinant leech-derived tryptase inhibitor in complex with trypsin - Implications for the structure of human mast cell tryptase and its inhibition. J Biol Chem 1997, 272:19931-19937.

55. Grzesiak A, Helland R, Smalas AO, Krowarsch D, Dadlez M, Otlewski J: Substitutions at the P-1 ' position in BPTI strongly affect the association energy with serine proteinases. J Mol Biol 2000, 301:205-217.

56. Berkmen M: Production of disulfide-bonded proteins in Escherichia coli. Protein Expr Purif 2012, 82:240-251.

57. Lefebvre J, Boileau G, Manjunath P: Recombinant expression and affinity purification of a novel epididymal human sperm-binding protein, BSPH1. Mol Hum Reprod 2009, 15:105-114.

58. Tait AR, Straus SK: Overexpression and purification of U24 from human herpesvirus type- 6 in E. coli: unconventional use of oxidizing environments with a maltose binding protein-hexahistine dual tag to enhance membrane protein yield. Microb Cell Fact 2011, 10:51. 
59. Saaranen MJ, Ruddock LW: Disulfide bond formation in the cytoplasm. Antioxid Redox Signal 2012. in press.

60. Xiong S, Wang YF, Ren XR, Li B, Zhang MY, Luo Y, Zhang L, Xie QL, Su KY: Solubility of disulfide-bonded proteins in the cytoplasm of Escherichia coli and its "oxidizing" mutant. World J Gastroenterol 2005, 11:1077-1082.

61. Hatahet F, Nguyen VD, Salo KE, Ruddock LW: Disruption of reducing pathways is not essential for efficient disulfide bond formation in the cytoplasm of E. coli. Microb Cell Fact 2010, 9:67.

62. Nguyen VD, Hatahet F, Salo KE, Enlund E, Zhang C, Ruddock LW: Preexpression of a sulfhydryl oxidase significantly increases the yields of eukaryotic disulfide bond containing proteins expressed in the cytoplasm of E.coli. Microb Cell Fact 2011, 10:1.

63. Tedford HW, Fletcher Jl, King GF: Functional significance of the betahairpin in the insecticidal neurotoxin omega-atracotoxin-Hv1a. J Biol Chem 2001, 276:26568-26576.

64. Mac TT, Beyermann M, Pires JR, Schmieder P, Oschkinat H: High yield expression and purification of isotopically labelled human endothelin-1 for use in NMR studies. Protein Expr Purif 2006, 48:253-260.

65. Abskharon RN, Ramboarina S, El Hassan H, Gad W, Apostol MI, Giachin G, Legname G, Steyaert J, Messens J, Soror SH, Wohlkonig A: A novel expression system for production of soluble prion proteins in E. coli. Microb Cell Fact 2012, 11:6.

66. Beld J, Woycechowsky KJ, Hilvert D: Small-molecule diselenides catalyze oxidative protein folding in vivo. ACS Chem Biol 2010, 5:177-182.

67. Contrepois K, Ezan E, Mann C, Fenaille F: Ultra-high performance liquid chromatography-mass spectrometry for the fast profiling of histone post-translational modifications.J J Proteome Res 2010, 9:5501-5509.

68. Horovitz A, Levitzki A: An accurate method for determination of receptorligand and enzyme-inhibitor dissociation constants from displacement curves. Proc Natl Acad Sci USA 1987, 84:6654-6658.

doi:10.1186/1475-2859-12-37

Cite this article as: Nozach et al: High throughput screening identifies disulfide isomerase DsbC as a very efficient partner for recombinant expression of small disulfide-rich proteins in E. coli. Microbial Cell Factories 2013 12:37.

\section{Submit your next manuscript to BioMed Central and take full advantage of:}

- Convenient online submission

- Thorough peer review

- No space constraints or color figure charges

- Immediate publication on acceptance

- Inclusion in PubMed, CAS, Scopus and Google Scholar

- Research which is freely available for redistribution 